International Journal of Life Sciences
Available online at www.sciencescholar.us
Vol. 4 No. 1, April 2020, pages: 78-86
e-ISSN: 2550-6986, p-ISSN: 2550-6994
https://doi.org/10.29332/ijls.v4n1.394

\title{
Traditional knowledge using medicinal plants in portoviejo canton
}

\author{
(

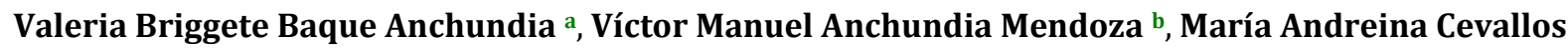 \\ Piloso ${ }^{c}$, Milton Manuel Vega Játiva ${ }^{\mathrm{d}}$
}

Manuscript submitted: 09 November 2019 Manuscript revised: 18 December 2019, Accepted for publication: 31 January 2020

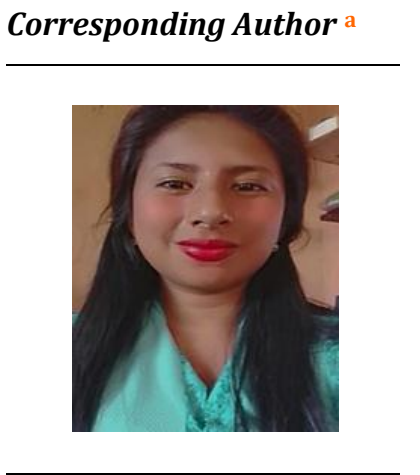

\section{Keywords}

inhabitant;

knowledge;

medicine;

plants;

recovery;

\begin{abstract}
The problem centers on the loss of empirical knowledge of the use of medicinal plants as a source of alternative medicine. The objective of the work is to carry out a study aimed at establishing the current state of local knowledge about the use of medicinal plants in rural parishes and 1 urban one of the Portoviejo canton. The method was based on the literature review on the subject and as techniques, the population survey was used on the knowledge and importance of medicinal plants. The main results of the research included the recovery of the use of medicinal plants as an alternative medicine use. As a result, it was obtained that the inhabitants of these parishes of average age between 35-59 years, had a high impact on the applied surveys of $39.1 \%$ of the use of medicinal plants and that the lowest ignorance of the use of medicinal plants as an alternative in medicine it was obtained by people of average age between 60 and over, but this is due to memory loss. It is concluded that knowledge about medicinal plants is regular since their inhabitants do not maintain continuity in their use.
\end{abstract}

\section{Contents}

Abstract

1 Introduction...

2 Materials and Methods

3 Results and Discussions

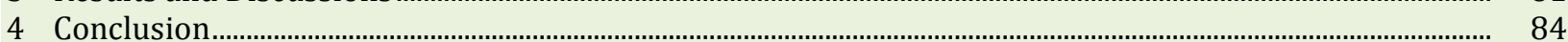

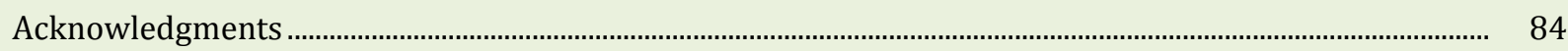

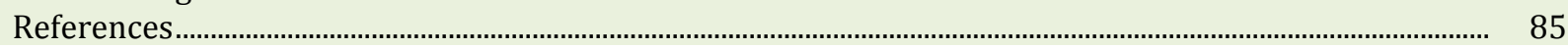

${ }^{a}$ Instituto Superior Tecnológico Paulo Emilio Macías, Portoviejo, Ecuador

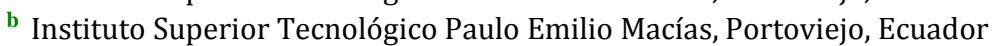

c Instituto Superior Tecnológico Paulo Emilio Macías, Portoviejo, Ecuador

d Instituto Superior Tecnológico Paulo Emilio Macías, Portoviejo, Ecuador 


\section{Introduction}

This paper highlights the different knowledge about the use of medicinal plants in the rural area of Portoviejo. The objective of the work is to conduct a study aimed at establishing the current state of local knowledge about the use of medicinal plants in rural parishes and 1 urban one of the Portoviejo canton, Manabí Province, Ecuador. Knowledge was also identified depending on the age of the inhabitants, comparing the knowledge of the use of medicinal plants in rural and urban parishes, as well as the type of gender and age, and finally determining which are the plants most used by the inhabitants for their ailments (Ljupco \& Jasminka, 2017; Zambrano et al., 2019).

According to Butler (2013), one of the most exciting research areas in the rainforests is ethnobotany, which is the study of how people use plants to treat ailments and diseases. Many native people in tropical areas have great knowledge about medicinal plants, which they frequently use as an alternative to modern medicines that are more expensive and difficult to obtain. To date, many of the medicines used in the western world have been derived from plants. Seventy percent of the plants identified as having anti-cancer characteristics according to the National Cancer Institute in the United States comes from forests.

According to the interview of Mejia (2003), he argues that traditional medicine (MT) is the pillar of modern medicine, conventional medicine is the consequence of accumulated knowledge in the development of thousands of years of popular experiences in which Acquired knowledge was taken advantage of by botanical doctors of modern schools.

With the investigation of Canales et al. (2006), Indigenous communities have a deep understanding of their environment and their ecology, they know of numerous uses that can be given to plants, for example, as medicines. This knowledge constitutes an important basis for the conservation of global biodiversity and for its sustainable use. Healing plants are the therapeutic resource par excellence of traditional Mexican medicine, which in large part is still salvageable and can be an important element to implement new health plans that combine popular knowledge with the scientist.

According to the report of Mancera (2015), medicinal plants have an important contribution to the health system of local communities, since they are used frequently by the majority of rural populations. Ecuador, due to its high biological and cultural diversity, has become one of the countries with great potential in relation to traditional medicine, so it is important to establish different aspects of importance, such as the ways of use of each plant and the benefits healing offered by traditional medicine in the different communities of the country.

According to Rios \& Koziol (2007), the use of plants in the various human populations of Ecuador is intrinsically linked to cultural traditions, so that ethnobotany has been key to investigate how each population takes advantage of the flora of their environment for being part substantial identity. This socio-environmental link demonstrates why accelerated acculturation processes and the disappearance of forests would result in not only the loss of useful plant species but also the ancient knowledge of indigenous peoples associated with their use.

According to Almeida (2000), at present, a large percentage of the world's population, particularly in developing countries, use plants to meet the primary needs of medical care, plants have played a fundamental role in the development of Andean cultures, being used as a source of food, medicines, fuel, building materials, tools and even an important place in their belief system and rites.

According to the report of Jorgensen \& Yanes (1999), Ecuador is considered one of the countries with the greatest biodiversity in the world, which gives it great potential in the use of plants, as a source of traditional medicine, being important to establish aspects, as the forms of use and the healing benefits they offer, in the different communities of the country. In this sense, ethnobotany is a useful tool for rescuing knowledge about the use of the plant resource and the interactions established between man and plants, over time.

According to the research of Muriel et al. (2006), there is important information on the traditional uses of Andean plant species provided in publications focusing on: 1. ornamental plants of Ambato 2. Taxonomic groups such as the Ciperáceas and Poáceas with its uses as fodder, medicinal, construction materials or

Anchundia, V. B. B., Mendoza, V. M. A., Piloso, M. A. C., \& Játiva, M. M. V. (2020). Traditional knowledge using medicinal plants in Portoviejo Canton. International Journal of Life Sciences, 4(1), 78-86. 
fillings, basketry and development of musical and hunting instruments 3 . Tree and shrub species found in remnants of Andean forests of the Ecuadorian northern highlands between 2,200-3,800 m.

According to the magazine article (Gallegos \& Gallegos, 2017), the various practices of traditional medicine developed throughout the world have contributed greatly to human health, particularly as providers of primary health care at the community level, which is why the World Health Organization (WHO) considers it as' the main pillar of the provision of health services, or its complement.

According to research by Garzón (2016), Traditional medicine is also considered as a complex system consisting of elements such as knowledge, traditions, practices, and beliefs, which are organized in an organized way through their own knowledgeable agents (specialist therapists, midwives, promoters, leftovers). These have their own method of diagnosis and treatment; as well as own therapeutic resources, which are recognized as medicinal plants.

According to Arenas (2008), there are data from the World Health Organization, $80 \%$ of the world's population uses traditional medicine to meet their primary medical assistance needs (WHO, 1987). These therapies are based primarily on the direct consumption of plants, so their study is a priority objective for both the World Health Organization and numerous international institutions.

In the investigation of Toscano (2006), health problems and the difficult achievement of commercial medicines have led again to the search for traditional medicine through the use and management of plants. The use of traditional medicine in the treatment of diseases is a practice that has been carried out since ancient times. These activities have undergone profound transformations and the values that the population has in relation to plants are disappearing with dizzying speed.

In the article by Carrillo et al. (2007), The use of plants as therapeutic agents in primary health care has been maintained over time and it can be said that approximately $60-80 \%$ of the world's population still depends largely on the traditional treatments that involve use of plant extracts or their active ingredients. According to Buenaño \& Mancera (2015), Medicinal plants have an important contribution to the health system of local communities, since they are used frequently by most rural populations. Ecuador, due to its high biological and cultural diversity, has become one of the countries with great potential in relation to traditional medicine, so it is important to establish different aspects of importance, such as the ways of use of each plant and the benefits healing offered by traditional medicine in the different communities of the country.

According to the research of De la Torre et al. (2008), in Ecuador, the use of medicinal plants is immersed in the daily lives of its inhabitants. Popular medicine is practiced mainly by inhabitants of rural areas, but also by citizens of all social classes. You can find a variety of plants with medicinal uses that are sold in markets in the Sierra, Costa and Amazon.

In the investigation Yesid et al. (2011), medicinal plants, which are all those wild, semi-wild, cultivated or managed species that are used in the country for their properties in the treatment or prevention of pathologies in people or animals, and are used as medications The active ingredients give these plants the medicinal quality and, consequently, the differential characteristic is their ability to counteract the effects of the disease on living organisms, that is, to act as a medicine. Medicinal plants considered as carriers of substances (or active ingredients) constituting medicines must meet the requirements of quality, safety and efficacy according to international and national regulations. This regulation indicates that, in medicinal plants, a drug is a binomial plant (or a mixture of plants) plus the therapeutic indication, and that it has properties to prevent, diagnose, treat, relieve or cure diseases or ailments, or to affect functions bodily, or mental state.

According to research by Méndez \& Üurán (1997), The exploration ethnobotanical constitutes one of the first steps in the study of medicinal plants, since it allows to know the medicinal flora of a region, the forms of use and the conditions for which the different species are used, in addition to contributing to the general knowledge of the flora. According to the article by Gomez (2012), at present, there is a growing interest in alternative medicine for the cure of a number of conditions and diseases that affect humans so that research that aims at the cultivation, study and processing of medicinal plants for therapeutic purposes are considered Strategic and important.

According to the research of Vázquez et al. (2011), the knowledge and use of medicinal plants between men and women is differential. Likewise, traditional knowledge systems remain among the indigenous population of the communities studied despite the lack of recognition of their role in health care and prevention by the institutions. In the investigation of García de Alba et al. (2011), The use of alternative 
medicines such as medicinal plants and dietary supplements has been a traditional practice that has not fallen into disuse, it is estimated that $80 \%$ of the population The world depends on traditional herbal remedies and that at least 35,000 plant species have potential for medicinal use.

In the article by Arias (2009), the consumption and management of wild plants as medicine is part of the traditional knowledge of different human populations. At present, the coexistence of various health systems is a reality in almost all of today's societies. These systems offer a wide range of approaches, resources, costs, and benefits for individual and collective health, and it is the demand and use of these resources that have determined the multiple nature of health care.

\section{Materials and Methods}

Field research was applied to collect information from respondents on the knowledge of medicinal plants, through the application of a survey. Work was carried out based on the population of the Portoviejo canton that amounts to 223,086 inhabitants, from which a stratified random sample was taken, choosing the 7 rural and 1 urban parishes, as can be seen in table 1 .

Table 1

Population and sample

\begin{tabular}{|c|c|c|}
\hline Parishes & Population & Sample (\%) \\
\hline Crucita & 14,050 & 0.40 \\
\hline Río Chico & 11,757 & 0.34 \\
\hline Calderón & 14,164 & 0.41 \\
\hline Chirijos & 2,362 & 0.06 \\
\hline Pueblo Nuevo & 3,169 & 0.09 \\
\hline Alajuela & 3,754 & 0.10 \\
\hline San Plácido & 7,687 & 0.22 \\
\hline Portoviejo & 223,086 & 0.92 \\
\hline Total & 280,029 & 2.54 \\
\hline
\end{tabular}

\section{Results and Discussions}

In the surveys carried out on the population of various rural areas and 1 an urban one from the Portoviejo canton it was announced what the percentage of knowledge of medicinal plants is. In figure 1, details the knowledge that the population of Puerto Rico has about the use of medicinal plants.

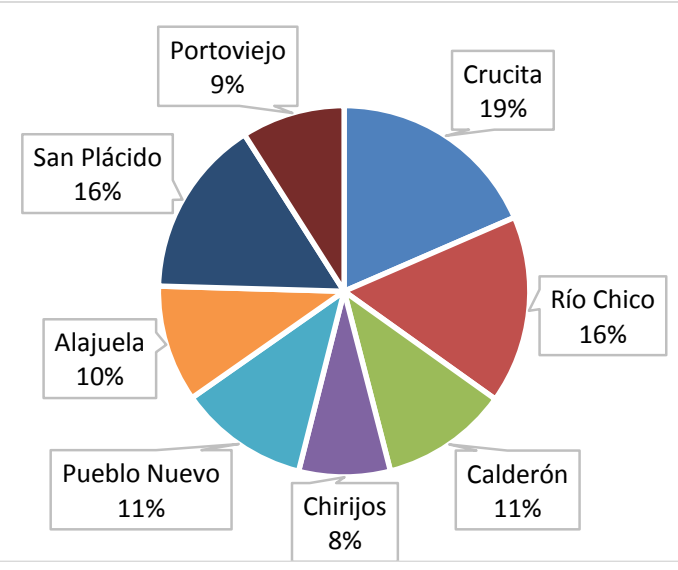

Figure 1. The knowledge that the population about the use of medicinal plants

Anchundia, V. B. B., Mendoza, V. M. A., Piloso, M. A. C., \& Játiva, M. M. V. (2020). Traditional knowledge using medicinal plants in Portoviejo Canton. International Journal of Life Sciences, 4(1), 78-86. 
As a result of the surveys applied in the 7 rural and 1 urban parishes of Portoviejo, 19\% of the population of the Crucita parish has knowledge of the use of medicinal plants; $16 \%$ of the population of Río Chico and San Plácido parishes, 11\% of Calderón and Pueblo Nuevo parishes, 10\% of Alajuela parish, 9\% of Portoviejo parish and $8 \%$ of Chirijos parish.

Figure 2 shows the provenance of the knowledge about the use of medicinal plants carried out by the population of Porto.

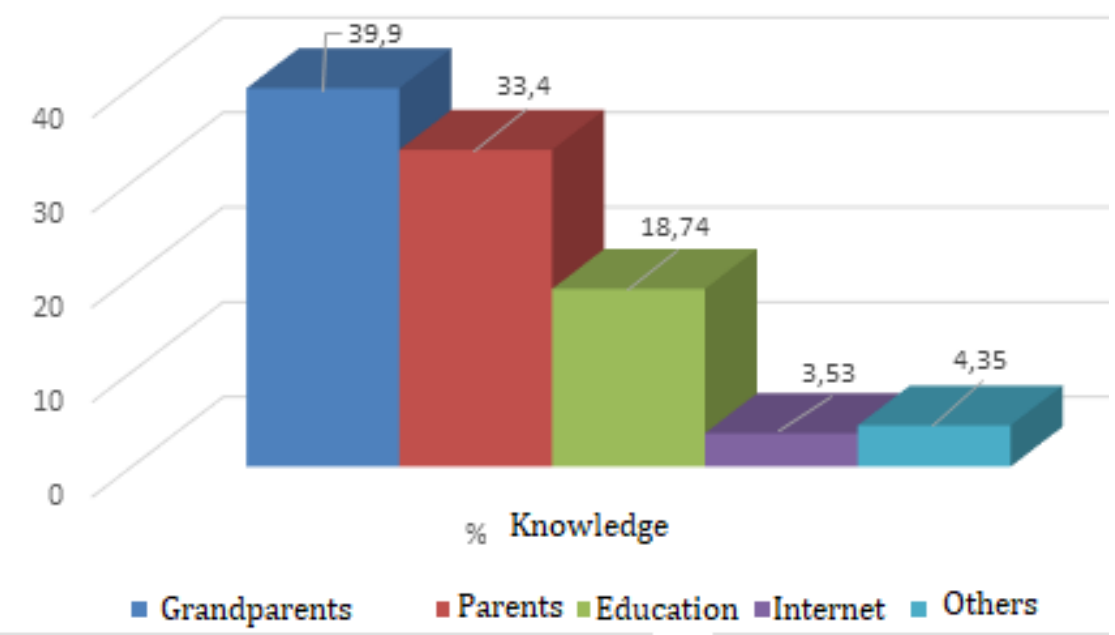

Figure 2. Origin of knowledge of the use of medicinal plants

According to the results, knowledge about the use of medicinal plants comes from grandparents in $39.9 \%$, from parents in $33.4 \%$, from school and college at $18.74 \%$ and internet at $3.53 \%$ and from other sources at $4.35 \%$

Figure 3 shows the medicinal plants most used by the rural and urban communities of the Portoviejo canton.

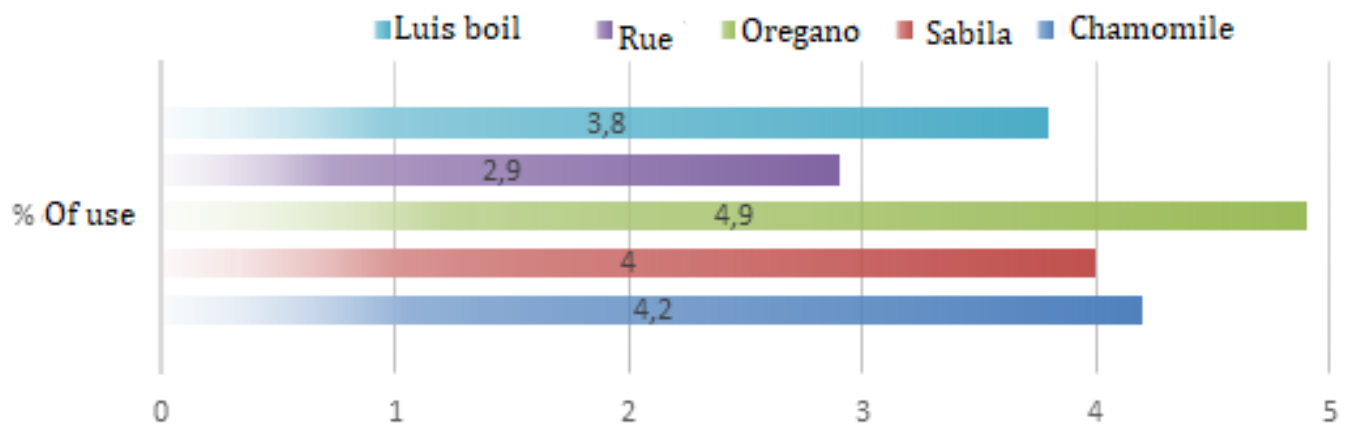

Figure 3. Most commonly used

Medicinal plants among the most commonly used medicinal plants, Luisa grass with $3.8 \%$, rue in $2.9 \%$, oregano in $4.9 \%$, and chamomile with $4.2 \%$ and finally aloe vera with $4 \%$.

Figure 4 shows the most common ailments treated with medicinal plants by the rural and urban communities of Portoviejo 


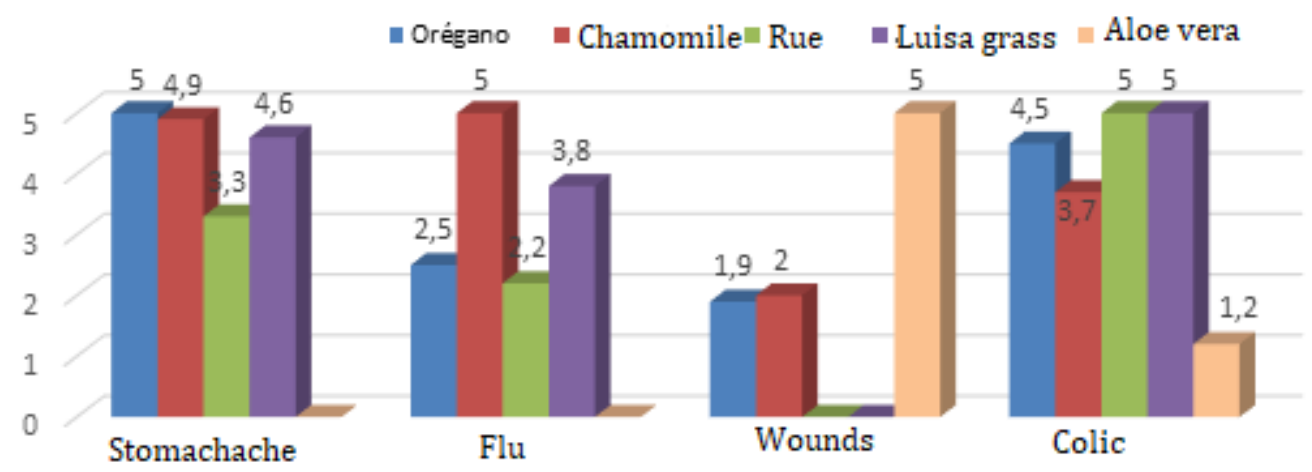

Figure 4. Most common ailments treated with medicinal plants

The results show that oregano is used for stomach aches in 5\%, in flu $2.5 \%$, in wounds in $1.9 \%$ and in colic $4.5 \%$ Chamomile is used a percentage of $4.9 \%$ in stomach pain, in flu with $5 \%$, in wounds with $2 \%$ and finally in colic with a percentage of 3.7\%. Rue is used in stomach pain using a percentage of 3.3\%, in influenza with $2.2 \%$, in wounds with $0 \%$ and finally in colic with a percentage of $5 \%$. Luisa herb is used in stomach pain using a percentage of $4.6 \%$, in flu with $3.8 \%$, in wounds with $0 \%$ and finally in colic with a percentage of $5 \%$. Aloe Vera is used to healing wounds by $5 \%$ and $1.2 \%$ to treat colic.

Figure 5 shows the medicinal plants that are known in the rural and urban areas of the Portoviejo canton

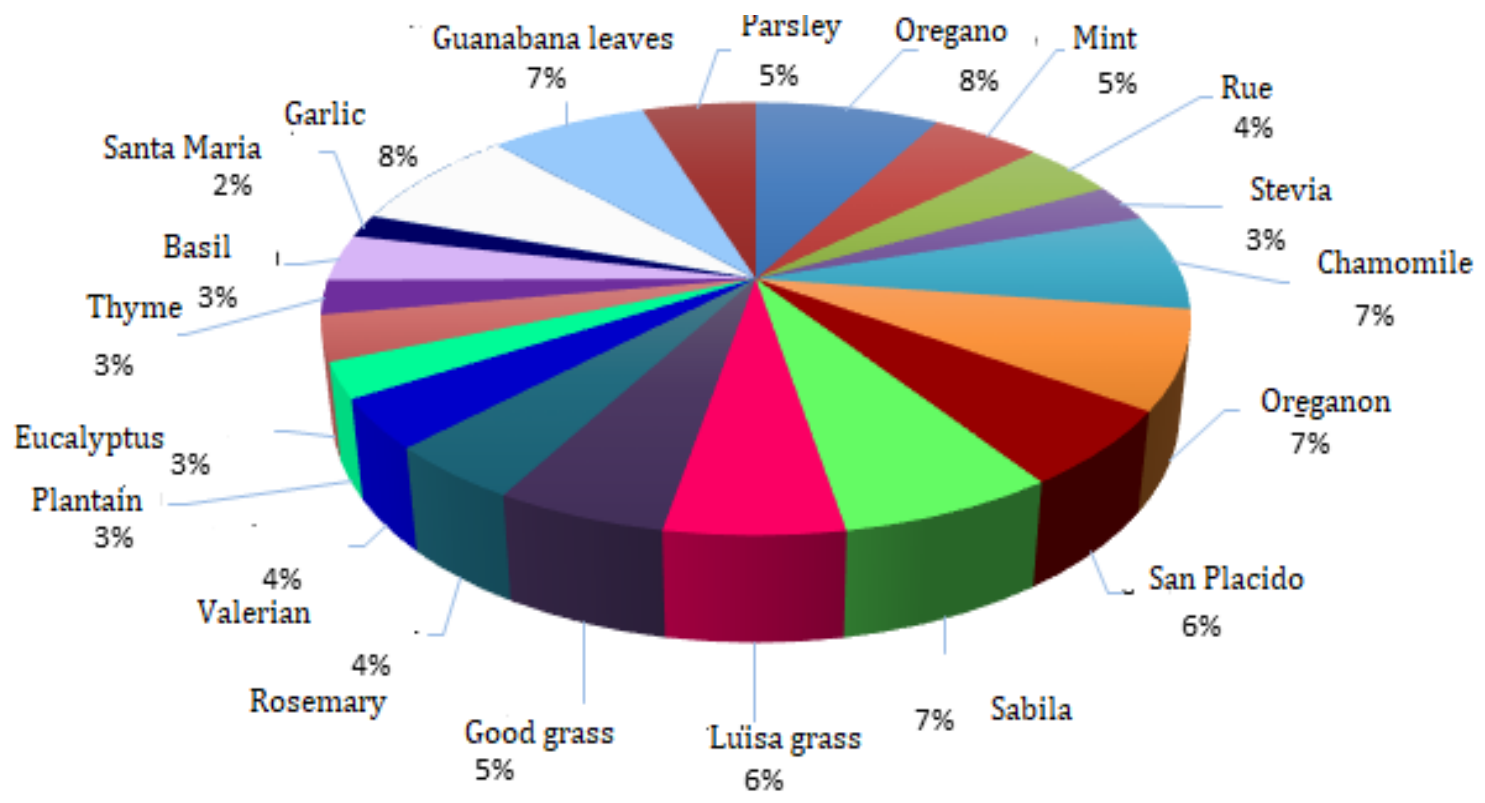

Figure 5. Species of medicinal plants best known

Within the medicinal plants best known by the inhabitants of the rural and urban communities of Portoviejo is oregano in $9.9 \%$, mint in $5.9 \%$, rue in $5 \%$, stevia in $3.4 \%$, chamomile in $8.7 \%$, oregano in $8.9 \%$, cinnamon in $7 \%$, aloe vera in $8.2 \%$, lemongrass in $6.8 \%$, good herb in $6.5 \%$, rosemary in $5.2 \%$, valerian in $4.5 \%$ plantain in $3.2 \%$, eucalyptus in $4.1 \%$, thyme in a $3.1 \%$, basil in $4.2 \%$, Santa Maria in $2.1 \%$, garlic in $9.4 \%$, soursop leaves in $8.5 \%$, and parsley in $6.1 \%$.

Anchundia, V. B. B., Mendoza, V. M. A., Piloso, M. A. C., \& Játiva, M. M. V. (2020). Traditional knowledge using medicinal plants in Portoviejo Canton. International Journal of Life Sciences, 4(1), 78-86. 


\section{Conclusion}

The knowledge of the population of Portoviejense about the use of medicinal plants is limited to a low percentage of the population. Medicinal plants are used mostly to cure ailments such as stomach pain, flu, colic, respiratory problems, skin inflammation, among others. This knowledge comes mostly from grandparents. Among the most used are oregano, rue, chamomile, aloe and lemon verbena.

\section{Acknowledgments}

A huge and very special thanks to Ing. Telly Yarita Macías Zambrano, Ms. Sc., a Career teacher and unconditional friend, who has been able to motivate us and guide us in the way of research, and lead us to an Institutional Recognition in the III Contest CREATE INGENIOS 2019 ISTPEM.

To our beloved parents, to the research tutor, Ing. Milton Veja Játiva, for his right direction in the investigation process, to fellow Alejandro Bravo, Tania Cevallos, Axel Chilán, Stefanía Mendoza, Milko Marcillo, Katherine Burgos, who kindly helped us to carry out the surveys in the different rural and urban parishes of the Portoviejo canton.

To the authorities of the Rector of the Institution, Lcda. Blanca Celina Meza Cruz, to the Academic ViceChancellor, Mgs. Gregorio Mendoza Cedeño, Mgs., And, for his constant support, for the Recognition we receive from him, by instituting the graduation modality with the publication of the scientific article.

To the editorial group of the IJLS Magazine for considering this research work for publication, so that this ancestral knowledge of the Montuvia manabita culture is not lost, on the contrary, it reaches the knowledge of many people throughout the planet. 


\section{References}

Almeida, E. (2000). Prehispanic cultures of Ecuador. Quito: Chasquiguiñá trips.

Arenas, P. (2008). Traditional botanical knowledge, commercial circulation and consumption of medicinal plants in an urban area of Argentina. silver: Applied Ethnobotany and Botany Laboratory (LEBA).

Arias, B. (2009). Diversity of uses, collection practices and differences according to gender and age in the use of medicinal plants in Córdoba, Argentina. Cordoba: BLACPMA ISSN 07177917.

Buenaño, M., \& Mancera, N. (2015). Ethnobotanical study of medicinal plants used by the inhabitants of the rural area of San Carlos Parish, Quevedo, Ecuador. Quevedo .: University and Health.

Butler, R. (2013). Tropical forests. Obtained from traditional knowledge of medicinal plants.

Canales, M., Hernandez, T., Caballero, J., Ramo, A., Duran, A., \& Lira, R. (2006). Quantitative analysis of the traditional knowledge of medicinal plants in San Rafael, Coxcatlán, Tehuacán-Cuicatlán Valley, Puebla, Mexico. Puebla: Mexican Botanical Act.

Carrillo, T., Moreno, R., \& Moreno, G. (2007). Importance of medicinal plants in health self-care in three hamlets of Santa Ana Trujillo, Venezuela. Famarcia faculty magazine 48(2), 22.

De la Torre, L., Alarcon, D., Peter, L., \& \& Salazar, J. (2008). Medicinal uses of plants. Quito: QCA Herbarium \& AAU Herbarium.

Gallegos, M., \& Gallegos, D. (2017). Medicinal plants used in the treatment of skin diseases in rural communities in the province of Los Ríos Ecuador. Scielo.

García de Alba, J., Ramirez, B., Robles, G., Zañudo, J., \& Salcedo, A. (2011). Knowledge and use of medicinal plants in the metropolitan area of Guadalajara. . Guadalajara: Contempt.

Garzon, L. (2016). Traditional knowledge about the medicinal plants of Yarumo (Cecropia sciadophylla), CARAMBOLO (Averrhoa carambola) and cat's claw (Uncaria tomentosa) In the indigenous shelter of Macedonia, Amazonas. SCielo, 10.

Gomez, R. (2012). Medicinal plants in a village in the state of Tabasco, Mexico. Rev. Fitotec. Mex. 35(1), 43-49.

Jorgensen, P., \& Yanez, L. (1999). Catalog of the Vascular Plants of Ecuador. Missouri Botanical Garden Press, USA

Ljupco, N., \& Jasminka, N. (2017). Unemployment an unauthorized unique road about apathy and poverty the Macedonian man without knowledge and skills. International Research Journal of Management, IT and Social Sciences, 4(4), 44-57.

Mancera, N. (2015). Ethnobotanical study of medicinal plants used by the inhabitants of the rural area of San Carlos Parish, Quevedo, Ecuador. Quevedo: Rev Univ. Health.

Mejia, A. (2003). Traditional knowledge about medicinal plants and their relationship with scientific knowledge. Seed magazine, 15.

Méndez, M., \& Üurán, R. (1997). Diagnosis of the current ethnobotanical knowledge of the medicinal plants of the yucatan peninsula. Yucatán: Scientific Research Center of Yucatán.

Muriel, P., De la Torre, L., \& Balslev, H. (2006). Ethnobotany in the Andes of Ecuador. San Andrés: Economic Botany of the Central Andes.

Rios, M., \& Koziol, M. (2007). Useful plants of Ecuador applications, challenges and pespertiva. Quito: Corporation for the Research and Monitoring Society of Ecuadorian Biodiversity (SIMBIOE).

Toscano, J. (2006). Traditional use of medicinal plants in la vereda san isidro, municipality of san josé de pareboyacá: a preliminary study using quantitative techniques. Bogotá: National University of Colombia Bogotá Headquarters.

Vázquez, B., Martínez, B., Fernández, A., \& Aguilar, A. (2011). Use and knowledge of medicinal plants by men and women in two indigenous locations in Coyomeapan, Puebla, Mexico. Caracas, Venezuela: Interciencia Association.

Yesid, H., García, H., \& Quevedo, G. (2011). Guidelines for the knowledge, conservation and sustainable use of native medicinal plants in colombia. Bogotá: Humboldt Institute.

Zambrano, T. Y. M., Vera, O. P. G., Plaza, C. L. M., \& Farfan, R. M. (2019). Recovery of ancestral knowledge of the use of leaf and bark towards royal ceibo. International Journal of Life Sciences,3(1), 41-47. https://doi.org/10.29332/ijls.v3n1.257

Anchundia, V. B. B., Mendoza, V. M. A., Piloso, M. A. C., \& Játiva, M. M. V. (2020). Traditional knowledge using medicinal plants in Portoviejo Canton. International Journal of Life Sciences, 4(1), 78-86. 


\section{Biography of Authors}

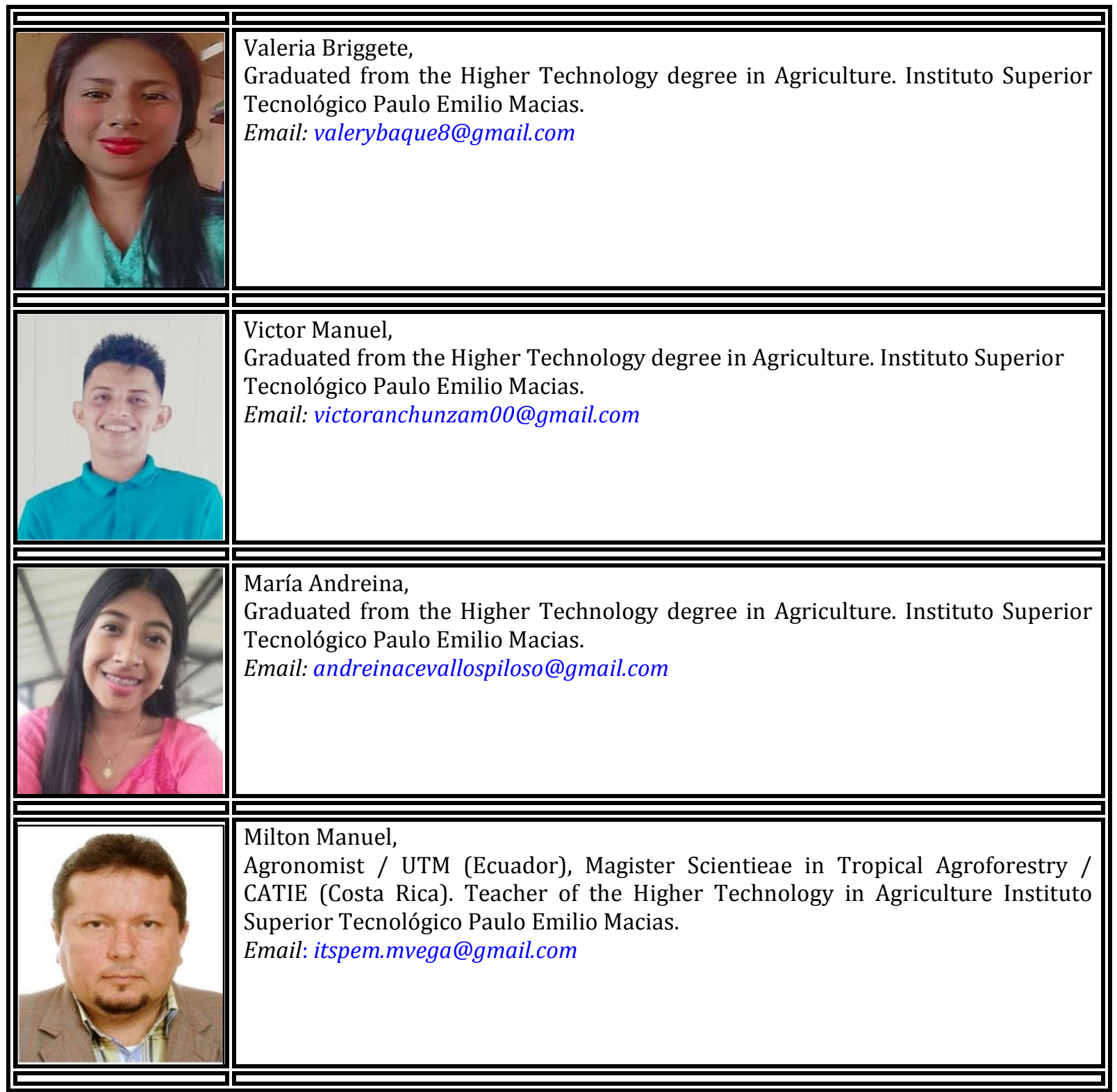

